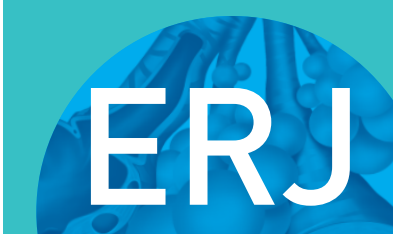

open research
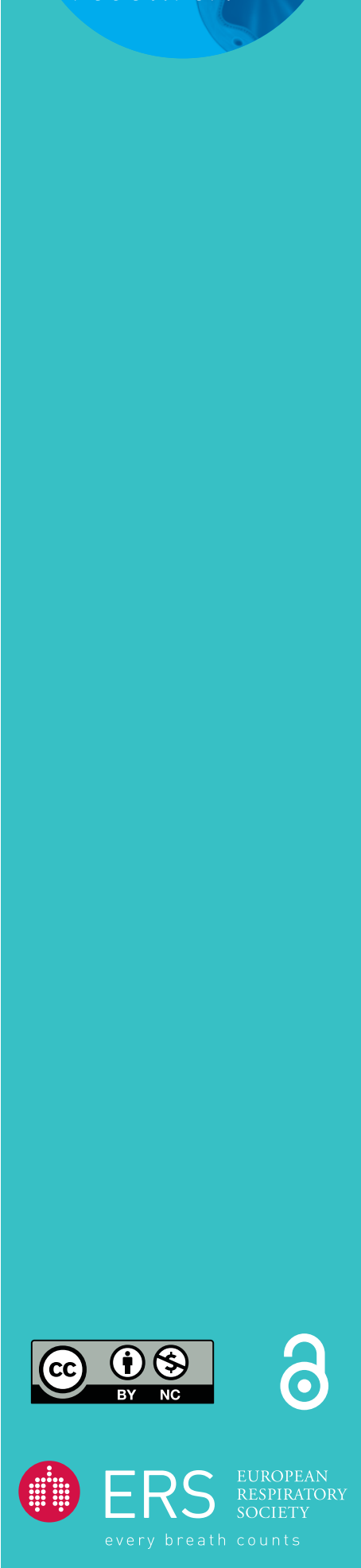

\section{Aetiology, diagnosis and treatment of moderate-to-severe haemoptysis in a North American academic centre}

\author{
Nicholas Quigley (), Sébastien Gagnon and Marc Fortin
}

Affiliation: Dept of Respiratory Medicine, Institut Universitaire de Cardiologie et de Pneumologie de Québec Université Laval (Québec Heart and Lung Institute), Québec City, QC, Canada.

Correspondence: Nicholas Quigley, IUCPQ, 2725, Chemin Ste-Foy, Québec City, QC, G1V 4G5, Canada. E-mail: nicholas.quigley.1 duulaval.ca

ABSTRACT Significant haemoptysis is a frightening event for patients and clinicians alike. There is a paucity of contemporary literature on the subject.

A retrospective analysis of hospitalisations for haemoptysis of more than $50 \mathrm{~mL} \cdot \mathrm{day}^{-1}$ in a tertiary referral centre during a 5-year period was performed. Patient's characteristics, haemoptysis aetiology, management and outcome were individually recorded. The aim of this study was to detail the causes of moderate $\left(50-200 \mathrm{~mL} \cdot \mathrm{day}^{-1}\right)$ to severe $\left(>200 \mathrm{~mL} \cdot \mathrm{day}^{-1}\right)$ haemoptysis along with the diagnostic measures and treatment options used in their management in a 21st century, tertiary-care North American centre.

A total of 165 hospitalisations for moderate-to-severe haemoptysis were included in the analysis. Lung cancer $(30.3 \%)$ and bronchiectasis $(27.9 \%)$ proved to be most frequent aetiologies. Computed tomography (CT) imaging and bronchoscopy were complementary in identifying the source of bleeding. Bronchial artery embolisation (BAE) was the most common treatment approach (61.8\%) and resulted in initial bleeding control in $73.5 \%$ of cases. In-hospital mortality was $13.9 \%$, varying from $3.3 \%$ in the moderate group to $24.7 \%$ in the severe group. Despite being the favoured approach in patients with more severe bleeding, initial BAE therapy was associated with a trend towards lower mortality compared to initial nonBAE therapy.

In summary, lung cancer and bronchiectasis were the main causes of moderate-to-severe haemoptysis in our population, CT and bronchoscopy are complementary in identifying the source of bleeding, bleeding volume is associated with outcomes and BAE is a key management tool.

@ERSpublications

In a contemporary North American population, lung cancer and bronchiectasis proved to be the leading causes of moderate-to-severe haemoptysis while CT and bronchoscopy appeared complementary in localising the source of bleeding https://bit.ly/2BFLvOT

Cite this article as: Quigley N, Gagnon S, Fortin M. Aetiology, diagnosis and treatment of moderate-to-severe haemoptysis in a North American academic centre. ERJ Open Res 2020; 6: 002042020 [https://doi.org/10.1183/23120541.00204-2020].

Received: 21 April 2020 | Accepted after revision: 24 July 2020

Copyright $\odot$ ERS 2020. This article is open access and distributed under the terms of the Creative Commons Attribution Non-Commercial Licence 4.0. 


\section{Introduction}

Moderate-to-severe haemoptysis, although a rare event, is a cause of great concern for both patients and physicians [1]. Urgent care is required as haemoptysis is associated with high rates of morbidity and mortality [2,3]. Previous studies have reported similar outcomes for moderate and severe haemoptysis [4].

Regarding the most frequent causes, there seems to be profound heterogeneity depending mainly on geographical location [5]. The aetiologies will also vary according to the severity of the haemoptysis. Tuberculosis (TB) has often been identified as one of the most common causes of significant haemoptysis in recent observational studies throughout the world [6]. However, this finding may not be accurate in some countries with higher socioeconomic levels [7]. Socioeconomic level may not be the only important factor as tuberculosis remains a frequent cause of significant haemoptysis in certain countries with a relatively high socioeconomic level $[3,8,9]$. Other frequent causes of moderate-to-severe haemoptysis reported are bronchiectasis, lung cancer, lower respiratory tract infections and mycetomas [6].

The value of different tests also remains unclear. Usually performed as the initial test, chest radiography identifies the location of bleeding in $45 \%$ to $65 \%$ of cases and leads to the identification of the cause of bleeding in as many as $35 \%$ of patients [10-13]. Whether a computed tomography (CT) scan or a bronchoscopy should be performed next depends on each individual case and if the patient's airway is secured. Chalumeau-Lemoine et al. [14] showed in a prospective study that flexible bronchoscopy and CT scan performed equally to localise the origin of bleeding, but that CT scan more frequently led to a definitive diagnosis.

Treatment of moderate-to-severe haemoptysis can differ significantly depending on the cause, but bronchial artery embolisation (BAE) has shown its effectiveness in a wide variety of situations since being first described in 1974 [15]. Various new endoscopic techniques used to control further bleeding have also provided interesting results [6].

Mortality rates for severe haemoptysis are historically reported to be exceeding $50 \%$ when not managed aggressively [8]. In recent cohorts of severe haemoptysis in France [3] and Singapore [16], in-hospital mortality rates between $6 \%$ and $13 \%$ were observed.

Considering the paucity of literature on moderate-to-severe haemoptysis, especially in the current North American setting, we conducted a retrospective observational study at our tertiary pulmonary medicine referral centre. Our objective was to provide contemporary epidemiological, diagnostic and therapeutic information.

\section{Methodology}

We performed a retrospective observational study of inpatients with a diagnosis of haemoptysis at our tertiary referral institution, the Québec Heart and Lung Institute (Québec City, Canada). After obtaining approval from the institution research ethics board (approval no. 2017-2835), we identified all hospitalisations with a diagnosis of haemoptysis during a 5-year-period from September 2011 to September 2016.

Two investigators (SG and NQ) manually reviewed all the charts to confirm the diagnosis of haemoptysis and to identify the episodes of moderate-to-severe haemoptysis. Episodes were categorised as mild, moderate or severe haemoptysis. Haemoptysis of $<50 \mathrm{~mL}$ per $24 \mathrm{~h}$ was classified as mild and patients were excluded. Haemoptysis of 50 to $200 \mathrm{~mL}$ per $24 \mathrm{~h}$ was classified as moderate and haemoptysis of $>200 \mathrm{~mL}$ per $24 \mathrm{~h}$ was classified as severe. Although there is no volume threshold consensus in the literature, we chose $50 \mathrm{~mL}$ and $200 \mathrm{~mL}$ per $24 \mathrm{~h}$ to define moderate and severe haemoptysis based on experience from previous reports [17-20].

Population baseline characteristics were collected. The volume, onset and duration of haemoptysis were noted as reported in the patient's chart. The aetiologies of the haemoptysis were classified using the following groups: lung cancer, bronchiectasis (including cystic fibrosis (CF)-associated), lower respiratory tract infections, pulmonary embolism, iatrogenic, idiopathic and others. For each haemoptysis episode, we reviewed the care process, including all diagnostic/imaging tests performed regarding their timing and ability to localise the source of bleeding. Also, we recorded the modalities of treatment used and the order in which they were used. If BAE was performed, we noted any recurrence of bleeding after embolisation. Need for intensive care unit (ICU) admission, endotracheal intubation and in-hospital mortality were also documented. Descriptive statistics were used and univariate analyses were performed. Fisher's exact test was used to compare binary variables due to small sample size while a t-test was used to compare continuous variables with a normal distribution. Statistical analyses were verified by an independent statistician who used SAS statistical analysis software. 


\section{Results}

A total of 560 hospitalisations with a diagnosis of haemoptysis were identified, 368 were excluded as they did not exceed $50 \mathrm{~mL}$ per $24 \mathrm{~h}$, and 27 others were excluded as they were recurrences in a patient already included in the study. Hence, 165 hospitalisations for moderate-to-severe haemoptysis were identified from September 2011 to September 2016. Baseline characteristics of the studied patients are summarised in table 1. We identified 73 (44.2\%) patients with severe haemoptysis and 92 (55.8\%) with moderate haemoptysis. The overall median bleeding volume was $187 \mathrm{~mL}$ per $24 \mathrm{~h}$. The most frequently encountered causes (table 2) were lung cancer (30.3\%), followed by bronchiectasis $(27.9 \%$; non- $\mathrm{CF}=21.2 \%, \mathrm{CF}=6.7 \%)$, lower respiratory tract infection $(4.2 \%)$, pulmonary embolism $(3.6 \%)$, iatrogenic $(3.6 \%)$ and arteriovenous malformation (AVM) (3.0\%). TB (1.2\%) was rarely the aetiology of moderate-to-severe haemoptysis in our population. The leading causes in the moderate and severe groups were respectively bronchiectasis and lung cancer. Despite thorough investigation, 20\% $(n=33)$ of haemoptysis remained cryptogenic. In this subgroup, post-discharge follow-up after $\geqslant 1$ year was available for 14 patients (42.4\%) and led to a retrospective diagnosis in only three patients (one lung cancer, one heart failure and one iatrogenic secondary to multiple antiplatelet medications). Among the 14 patients, 3 had recurrence of haemoptysis within a year following discharge.

Chest radiography was the most performed diagnostic test $(\mathrm{n}=152,92.1 \%)$ but led to localisation of bleeding source in only $13.8 \%$ of cases (21 of 152). Noncontrast CT imaging of the chest was obtained in 27 patients (16.4\%), while contrast-enhanced CT was performed in 92 patients $(55.8 \%)$. The bleeding localisation rates were $51.9 \%$ (14 of 27 ) and $52.2 \%$ (48 of 92), respectively. Flexible bronchoscopy, performed in 110 patients $(65.5 \%)$, was the most effective at localising the source of bleeding (72 of 110 , $65.5 \%)$. The source of haemoptysis remained unidentified in $27 \%(\mathrm{n}=45)$ of cases, including in 6 patients

\section{TABLE 1 Patient characteristics}

\section{Characteristics}

Subjects

\section{Patients $n$}

Female sex

Age years mean

Smoking

Active

Pack-years mean ${ }^{\#}$

Prior episode(s) of haemoptysis

Comorbidities

COPD

Bronchiectasis

Cystic fibrosis

$47(28.5 \%)$

$42(25.5 \%)$

Pulmonary hypertension

$13(7.9 \%)$

Atrial fibrillation

$7(4.2 \%)$

Cardiac ischaemic disease

$24(14.5 \%)$

Heart failure

$40(24.2 \%)$

Thromboembolic disease

$15(9.1 \%)$

Lung cancer

Past lung cancer

$6(3.6 \%)$

Active lung cancer

$13(7.9 \%)$

Known endobronchial lesion

$42(25.5 \%)$

Medication

ASA

$19(11.5 \%)$

Clopidogrel

$57(34.5 \%)$

ASA and clopidogrel

$7(4.2 \%)$

Oral anticoagulant

$5(3.0 \%)$

Apixaban/rivaroxaban/dabigatran

$8(4.8 \%)$

Warfarin

INR mean

$20(12.1 \%)$

LMWH (therapeutic dosage)

Intravenous heparin

$7(4.2 \%)$

ASA+one anticoagulant

Data are presented as $n(\%)$, unless otherwise stated. ASA: acetylsalicylic acid; INR: international normalised ratio; LMWH: low molecular weight heparin. ${ }^{\#}$ : including both active and former smokers. 


\begin{tabular}{|c|c|c|c|}
\hline & Moderate & Severe & Total \\
\hline Patients & $92(55.8 \%)$ & $73(44.2 \%)$ & $165(100 \%)$ \\
\hline \multicolumn{4}{|l|}{ Aetiologies } \\
\hline Lung cancer & $20(21.7 \%)$ & $30(41.7 \%)$ & $50(30.3 \%)$ \\
\hline Bronchiectasis & $24(26.1 \%)$ & $11(15.1 \%)$ & $35(21.2 \%)$ \\
\hline Idiopathic & $21(22.8 \%)$ & $12(16.4 \%)$ & $33(20.0 \%)$ \\
\hline Cystic fibrosis-related bronchiectasis & $5(5.4 \%)$ & $6(8.2 \%)$ & $11(6.7 \%)$ \\
\hline Lower respiratory tract infection & $5(5.4 \%)$ & $2(2.8 \%)$ & $7(4.2 \%)$ \\
\hline latrogenic & $5(5.4 \%)$ & $1(1.4 \%)$ & $6(3.6 \%)$ \\
\hline Pulmonary embolism & $4(4.4 \%)$ & $2(2.8 \%)$ & $6(3.6 \%)$ \\
\hline \multicolumn{4}{|l|}{ Others } \\
\hline Arteriovenous malformation & $2(2.2 \%)$ & $3(4.1 \%)$ & $5(3.0 \%)$ \\
\hline Pulmonary oedema & $2(2.2 \%)$ & $1(1.4 \%)$ & $3(1.8 \%)$ \\
\hline Aspergilloma & $1(1.1 \%)$ & $1(1.4 \%)$ & $2(1.2 \%)$ \\
\hline Tuberculosis & $1(1.1 \%)$ & $1(1.4 \%)$ & $2(1.2 \%)$ \\
\hline Vasculitis & $1(1.1 \%)$ & 0 & $1(0.6 \%)$ \\
\hline Arterio-bronchial fistula & 0 & $1(1.4 \%)$ & $1(0.6 \%)$ \\
\hline Granulomatous disease & 0 & $1(1.4 \%)$ & $1(0.6 \%)$ \\
\hline Lymphoma & 0 & $1(1.4 \%)$ & $1(0.6 \%)$ \\
\hline Pulmonary hypertension & $1(1.1 \%)$ & 0 & $1(0.6 \%)$ \\
\hline Median volume in $24 \mathrm{~h} \mathrm{~mL}$ & 98 & 316 & 187 \\
\hline \multicolumn{4}{|l|}{ Duration of active haemoptysis } \\
\hline$<8 \mathrm{~h}$ & $12(13.0 \%)$ & $18(24.7 \%)$ & $30(18.2 \%)$ \\
\hline $8-24 \mathrm{~h}$ & $20(21.7 \%)$ & $25(34.2 \%)$ & $45(27.3 \%)$ \\
\hline $24-48 h$ & $18(19.6 \%)$ & $9(12.3 \%)$ & $27(16.4 \%)$ \\
\hline$>48 \mathrm{~h}$ & $42(45.7 \%)$ & $21(28.9 \%)$ & $63(38.2 \%)$ \\
\hline
\end{tabular}

who decided to receive palliative care prior to completing investigations. Two patients died from uncontrolled bleeding prior to completing investigations.

Bleeding source was localised in 13 of 24 (54.2\%) patients who underwent CT imaging (including contrast-enhanced) without bronchoscopy and in 19 of 21 patients (90.5\%) in whom bronchoscopy was performed without additional CT scan. Eighty-nine patients underwent both CT imaging and bronchoscopy (figure 1). Among the 71 patients in whom the CT was carried out first, localisation of bleeding was established in 42 cases (59\%). Among the 29 patients in whom the CT performed first failed to localise the bleeding, bronchoscopy succeeded in 13 (45\%). Among the 18 patients in whom bronchoscopy was performed before CT, the bleeding source was identified in $13(72 \%)$. In the five patients in whom bleeding source remained unknown after bronchoscopy, CT imaging localised bleeding source in three cases $(60 \%)$.

BAE was the most common treatment approach $(n=102(61.8 \%)$ ) (figure 2). Eighty-seven patients were oriented towards BAE as an initial therapy and 15 others received it after other modalities failed. Surgery (five patients, $3 \%$ ) was only rarely used. Eight patients (4.8\%) had therapeutic bronchoscopy to control the haemoptysis. Seventy-four patients initially had a noninvasive observation approach (figure 3). Among them, 28 received antibiotics and 20 had their antiplatelet or anticoagulant medication stopped. Fifteen patients eventually needed rescue BAE after the initial approach failed, including 11 patients in the antibiotics subgroup and 3 patients in the antiplatelet or anticoagulation cessation subgroup. Thirteen patients died, 6 after a decision of palliative care was made, 5 of uncontrolled haemoptysis (including 1 after a failed BAE) and 2 from other causes.

Despite being the favoured approach (table 3) in patients with higher mean bleeding rate $(206.8 \mathrm{~mL}$ per $24 \mathrm{~h}$ versus $163.3 \mathrm{~mL}$ per $24 \mathrm{~h}$ ), initial BAE therapy was associated with a trend towards lower mortality compared to initial non-BAE therapy $(11.5 \%$ versus $17.9 \%, \mathrm{p}=0.27)$. However, in the initial non-BAE therapy group, four patients were oriented to palliative care upon admission and eventually died during their hospitalisation. If we exclude these 4 patients, mortality in the initial non-BAE therapy group is $13.5 \%$ (10 of 74$)$.

Haemoptysis recurrence rate prior to discharge after BAE was 26.5\% (27 of 102). Of those 27 patients, 10 (37.0\%) underwent a second BAE. A second BAE stopped the haemoptysis in all but two patients, who eventually died of uncontrolled bleeding. Among the 17 patients who did not have a second BAE, 


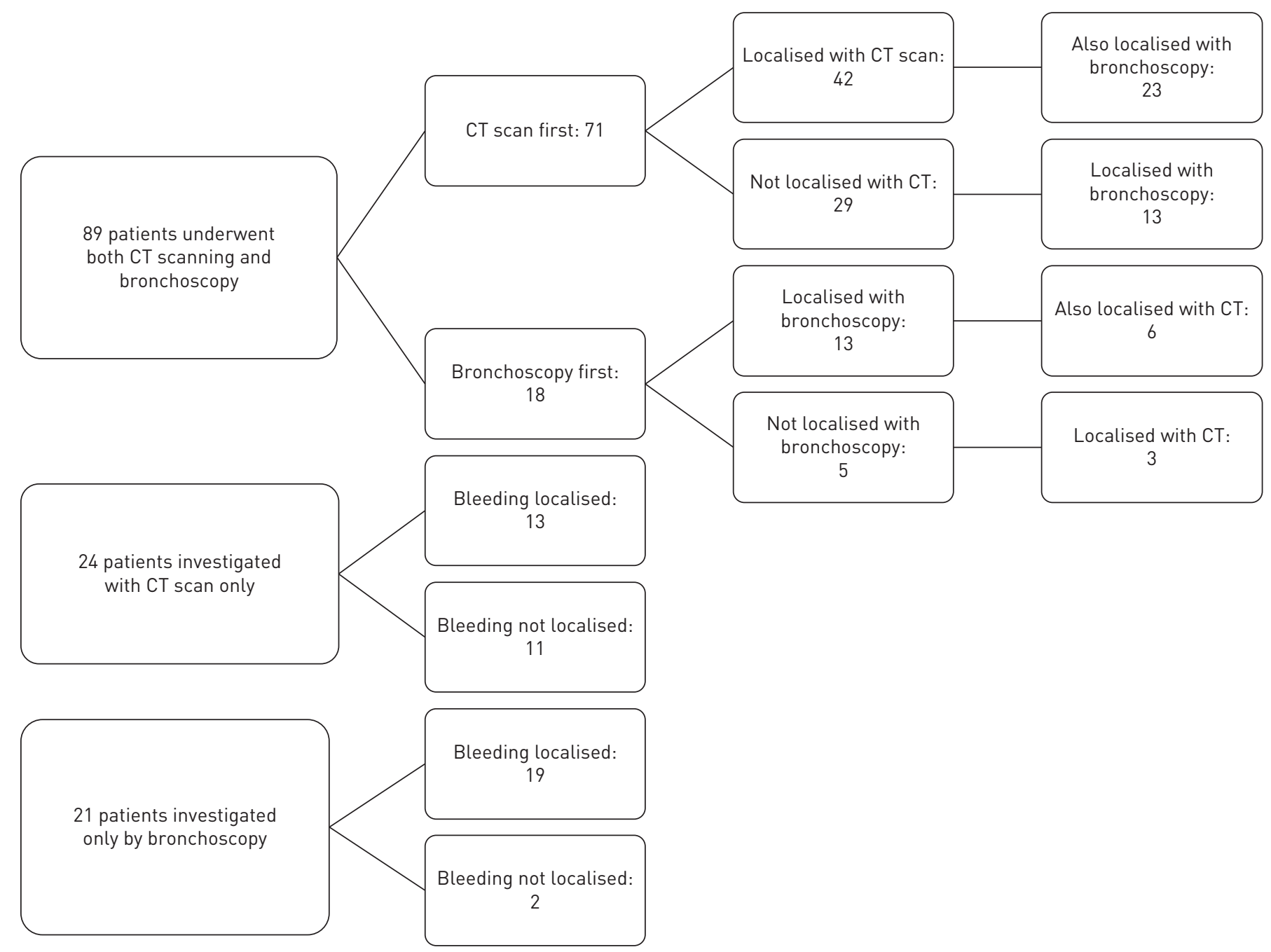

FIGURE 1 Bleeding localisation of bronchoscopy and computed tomography (CT).

observation alone led to bleeding cessation in 11, 3 died of uncontrolled bleeding, 2 opted for palliative care and 1 had endoscopic control of the haemoptysis.

The average length of hospital stay was 9.1 days. A total of 62 patients (38\%) were admitted to the ICU, where the mean stay was 3.2 days. Twenty-four individuals (14\%) were intubated and mechanically ventilated. The in-hospital mortality rate was $13.9 \%(n=23)$, mostly after changes in goals of care $(n=11,47.8 \%)$ and uncontrolled haemoptysis $(n=6,26.0 \%)$. Among those whose goals of care were changed, six opted for palliative care when faced with the underlying progression of their cancer and associated comorbidities and five patients were transferred to a palliative care unit after treatments failed to control the bleeding. Lung cancer (12 of $23,52.2 \%$ ) was the most frequent aetiology leading to death during the hospitalisation and mortality rate was higher when lung cancer was the underlying aetiology (24.0\% (12 of 50 ) versus $7.7 \%$ (11 of 142) for other causes).

Severe haemoptysis was associated with poor outcomes (table 4). The mean length of hospital stay was not statistically significantly longer (10.0 versus 8.4 days, $\mathrm{p}=0.47)$. However, ICU stay $(57.5 \%$ versus $21.7 \%$, $\mathrm{p}<0.001)$ and endotracheal intubation $(29.2 \%$ versus $3.3 \%, \mathrm{p}<0.001)$ were more likely to be needed. In-hospital mortality rate was also higher in those with severe haemoptysis $(27.4 \%$ versus $3.3 \%, \mathrm{p}<0.001)$.

\section{Discussion}

In this observational, retrospective study of cases of moderate and severe haemoptysis presenting to our referral centre, we identified causes of significant haemoptysis that were somewhat different from what is currently shown in various cohorts around the globe. To our knowledge, this is the largest modern moderate-to-severe haemoptysis cohort in a tertiary North American setting. In opposition to previous 


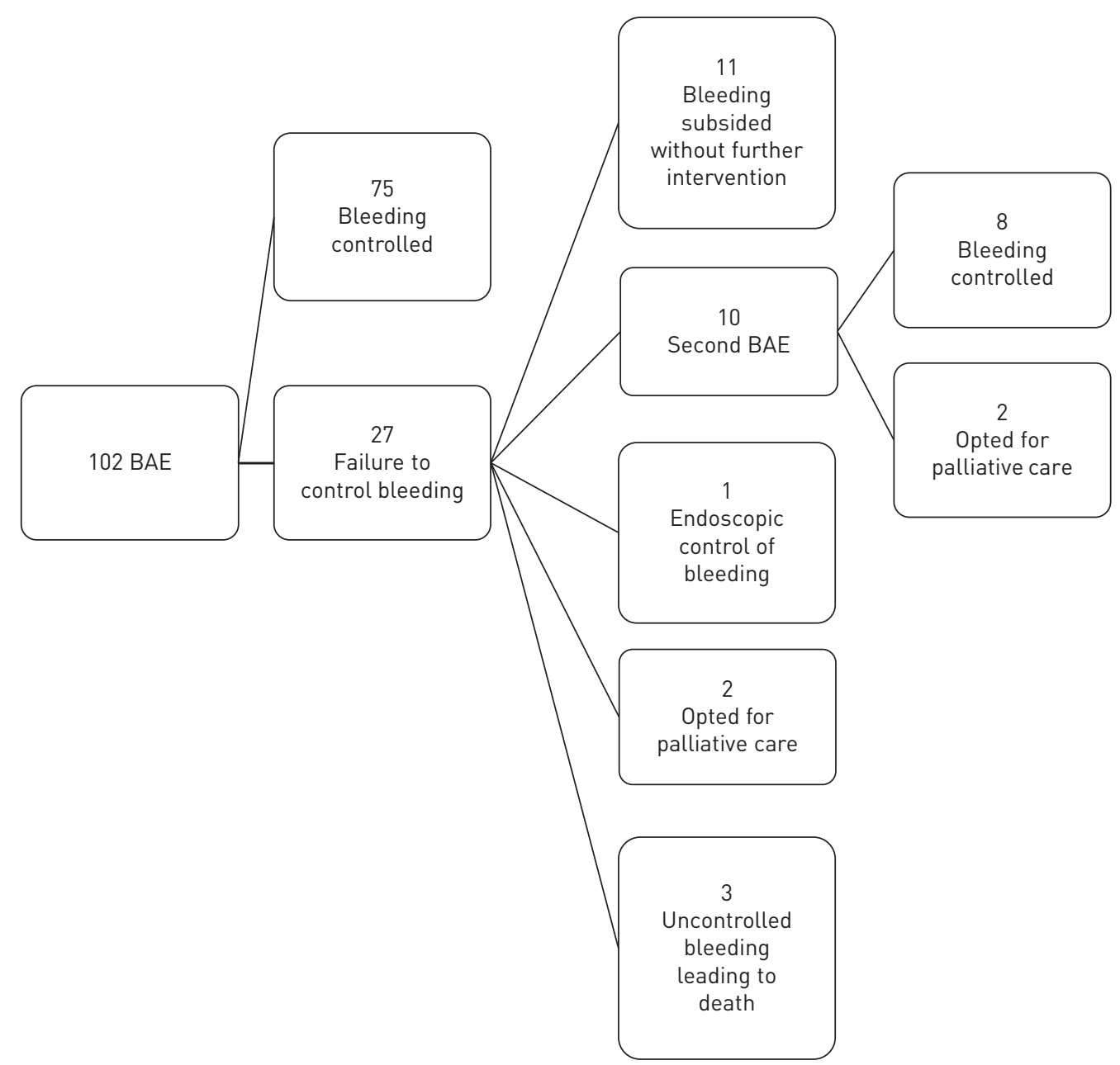

FIGURE 2 Outcomes of patients undergoing bronchial artery embolisation (BAE).

reports, including some from France [5, 8, 11], Austria [21] and the USA [7] in the years 2000-2010, TB was an uncommon cause of significant haemoptysis in our population; however, this lower rate of tuberculosis is to be interpreted in the context of the low prevalence of the disease in our catchment area. Lung cancer was the leading cause of significant haemoptysis in our cohort, especially in severe bleeding of more than $200 \mathrm{~mL}$ per $24 \mathrm{~h}$ (41.7\% of these cases). The proportion of lung cancer differs from other studies in higher socioeconomic settings where bronchiectasis was at the forefront and lung cancer was a less frequent aetiology, except in one series by VALIPOUR et al. [21] from Austria (35\% of lung cancer in haemoptysis of $>150 \mathrm{~mL}$ ). Furthermore, lung cancer was also the aetiology associated with the highest mortality rate of (24.0\% versus $7.7 \%$ for other causes). In both groups (moderate and severe), lung cancer, bronchiectasis and idiopathic were the most frequent diagnoses. The relatively significant number of cases due to CF-associated bronchiectasis is probably specific to our population and imputable to a reference bias at our institution which is the referral CF centre of a 2 million people catchment area with a relatively high prevalence of $\mathrm{CF}$ due to local genetic factors. A high proportion of haemoptysis remained of unknown aetiology (20\%) despite thorough investigation which is consistent with previous publications. ABdulmalaK et al. [5] extracted data from the French nationwide database over 5 years (2008-2012) and analysed 75000 cases of haemoptysis (mild to severe). A total of $50 \%$ of cases were cryptogenic. In more severe haemoptysis, KirAL et al. [22] identified 22\% of idiopathic cases in 203 Turkish patients with haemoptysis of $>200 \mathrm{~mL}$ per $24 \mathrm{~h}$. FARTOUKH et al. [8] in France noted 18\% of cryptogenic haemoptysis in their series of 1087 patients necessitating ICU admission.

In our series, flexible bronchoscopy was globally slightly superior to CT at localising the source of bleeding (65.5\% versus 52.0\%). Patients initial characteristics did not differ significantly between those who had bronchoscopy or CT first except that more patients initially undergoing flexible bronchoscopy had previously been intubated. The sequence of exam seems to have been frequently dictated by their 


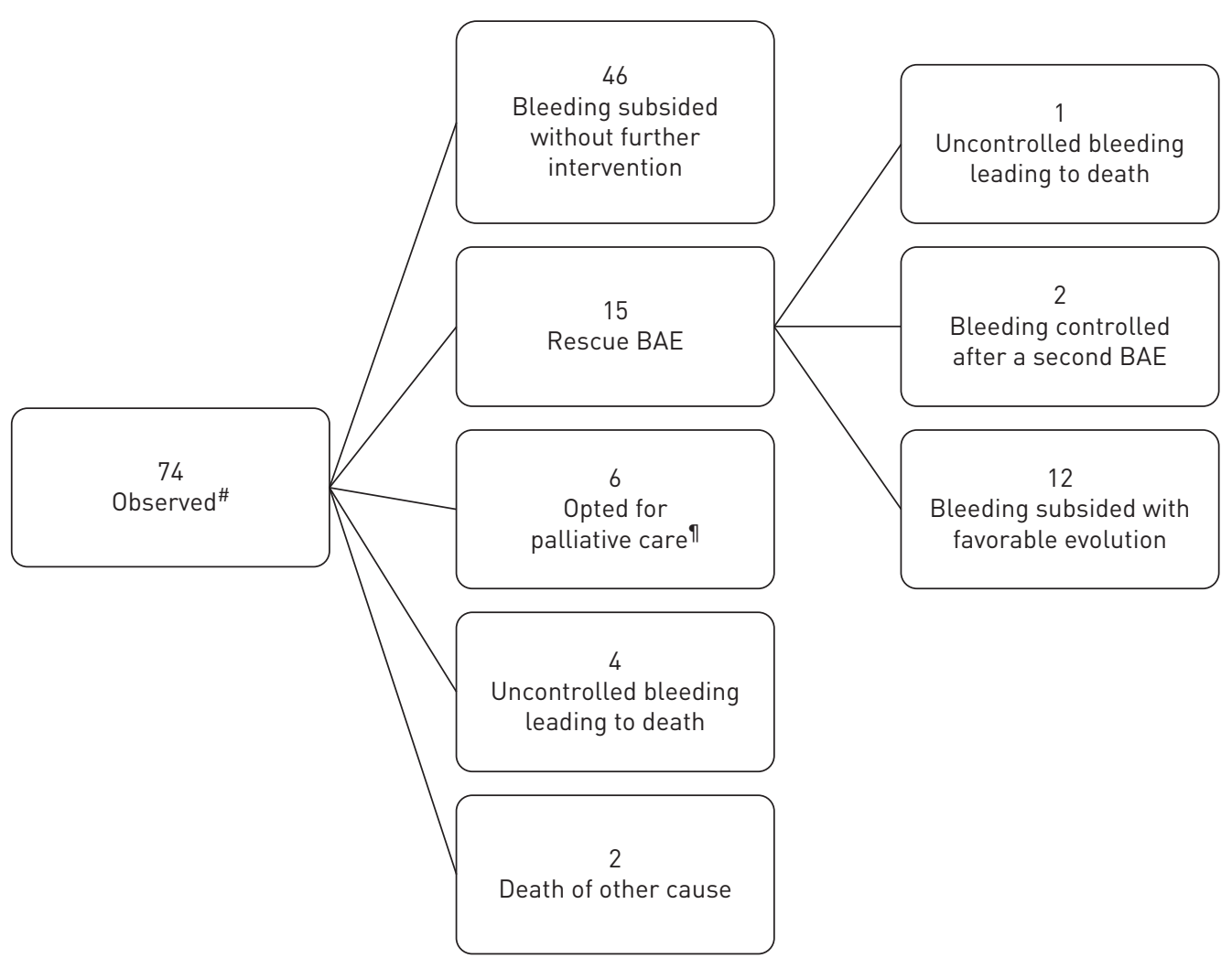

FIGURE 3 Outcomes of patients who were treated with a noninvasive observational approach. BAE: bronchial artery embolisation. \#: amongst observed patients, 28 were on antibiotics and 20 had their antiplatelets and/or anticoagulation stopped; " : six patients opted for palliative care with ongoing bleeding.

\section{TABLE 3 Initial bronchial artery embolisation (BAE) strategy versus non-BAE strategy}

Initial BAE strategy

Patients
Age years mean
Female sex
Volume of haemoptysis $\mathrm{mL}$ per $24 \mathrm{~h}$ mean
Severe haemoptysis
Length of hospital stay days mean
ICU
Endotracheal intubation
In-hospital mortality
Aetiologies
Lung cancer
Bronchiectasis
Idiopathic
Cystic fibrosis-related bronchiectasis
Lower respiratory tract infection
latrogenic
Pulmonary embolism
Arteriovenous malformation
Pulmonary oedema
Others

Patients

Age years mean

Volume of haemoptysis $\mathrm{mL}$ per $24 \mathrm{~h}$ mean

Severe haemoptysis

\section{ICU}

In-hospital mortality

tiologies

Lancer

Idiopathic

latrogenic

Arteriovenous malformation

Others
$87(52.7 \%)$
64.2
$31(35.6 \%)$
206.8
$47(54.0 \%)$
9.6
$40(46.0 \%)$
$15(17.2 \%)$
$10(11.5 \%)$
$27(31.0 \%)$
$17(19.5 \%)$
$23(26.4 \%)$
$5(5.7 \%)$
$1(1.1 \%)$
$3(3.4 \%)$
$1(1.1 \%)$
$4(4.6 \%)$
0
$6(6.9 \%)$

Initial non-BAE strategy"

ICU: intensive care unit. \# 15 patients initially assigned to non-BAE approach later received BAE. Eight had bronchiectasis (including one CF-related), four had lung cancer, one had iatrogenic haemoptysis, one had arteriovenous malformation and the other case remained idiopathic; ": four of those patients had surgery. 


\begin{tabular}{lccc}
\hline TABLE 4 Outcomes and mortality & & & \\
& Total $(\mathbf{n = 1 6 5 )}$ & Moderate $(\mathbf{n = 9 2 )}$ & Severe $(\mathbf{n}=\mathbf{7 3})$ \\
\hline $\begin{array}{l}\text { Number of days in hospital mean } \\
\text { ICU admission }\end{array}$ & 9.1 & 8.4 & 10.0 \\
Number of days in ICU median & $62(38 \%)$ & $20(21.7 \%)$ & $42(57.5 \%)$ \\
$\begin{array}{l}\text { Endotracheal intubation } \\
\text { In-hospital mortality }\end{array}$ & $24(14.2 \%)$ & 3.5 & 3.0 \\
& $23(13.9 \%)$ & $3(3.3 \%)$ & $21(29.2 \%)$ \\
ICU: intensive care unit. & & $3.3 \%)$ & $20(27.4 \%)$ \\
\hline
\end{tabular}

availability as a CT had been ordered and was pending in the vast majority of patients undergoing bronchoscopy first. Adding bronchoscopy after CT and CT after bronchoscopy both seem to have added localisation value. Based on our data, we cannot recommend one localising exam over the other, but we believe that both exams are complementary. CT scanning is the exam of choice for all noncentral lesions and for identifying bronchiectasis, peripheral lung cancer, pulmonary embolism, arteriovenous malformation and aspergillomas [23]. On the other hand, bronchoscopy is valuable for more central or endobronchial lesions, where it may allow concurrent identification, histopathological diagnosis and therapeutic measures on a specific bleeding site. We recommend, based on our experience, a contrast-enhanced CT with a dedicated arterial phase first if the patient is stable enough to be sent to radiology. This will provide localisation information in a significant proportion of patients, clarify anatomy and define bronchial or pulmonary vasculature origin for BAE if it is deemed necessary. If, after the CT, the bleeding source remains unidentified, we believe flexible bronchoscopy, if clinically judged safe, has an interesting additional value. It is important to understand that we focused on localisation rate, but exams also have a diagnostic rate which was felt to be too difficult to evaluate retrospectively as CT is often suggestive but not diagnostic.

The safety of a flexible bronchoscopy in patients presenting significant haemoptysis is of the utmost importance. While no specific criteria defining safety other than clinician judgment was highlighted by this retrospective study, we believe that bronchoscopy should not be performed in an actively bleeding patient unless a plan and appropriate material are prepared for eventual endotracheal intubation. Furthermore, endobronchial therapies to intervene on the source of bleeding such as cold saline, adrenaline or tranexamic acid should be readily available. Ideally, CT scanning should be performed first (if the patient is stable enough to be sent to the radiology department) and bronchoscopy kept for later as the patient's condition stabilises and CT images allow for guidance of the endoscopic technique. Bronchoscopy under general anaesthesia may be necessary when high volume active bleeding and hypoxaemia are concerns. Rigid bronchoscopy can be useful in this population by stabilising the airway, providing the possibility to use large bore suction and allowing endoscopic interventions such as the use of laser or argon and the positioning of oxidised regenerated cellulose [21], biocompatible glue [24], endobronchial stents [25] or silicone spigots [26, 27].

Regarding treatment options, BAE was the main treatment used in moderate-to-severe haemoptysis and achieved a relatively high bleeding control rate. It is interesting to note that there was a trend towards lower mortality with initial BAE approach, despite being the favoured therapy for more severe bleeding.

When designing this study, we chose thresholds of 50 and $200 \mathrm{~mL}$ per $24 \mathrm{~h}$ for moderate and severe haemoptysis based on reports from previous studies [17-20]. As mentioned earlier, aetiologies were globally similar between the two groups, but outcomes were different. Severe haemoptysis was associated with a higher mortality rate and a greater need for endotracheal intubation and ICU. The overall in-hospital mortality rate of $13.9 \%$ is higher than the 6.5\% reported by FARTOUKH et al. [3] in 1087 patients who needed ICU admission in France. The mortality rate of $27.4 \%$ in severe cases is also much higher than the $12.9 \%$ rate reported by ONG and ENG [16] in a population from Singapore with haemoptysis of $>300 \mathrm{~mL} \cdot \mathrm{day}^{-1}$ or necessitating intubation. The higher proportion of patients with lung cancer most likely account for the difference (lung cancer was the aetiology in 17\% [3] and 7\% [16] of patients in the previously mentioned studies). Had we chosen different thresholds for moderate and severe haemoptysis; mortality rates would not have been dramatically different. Had we opted to identify severe bleeding as $100,150,200,250$ or $350 \mathrm{~mL} \cdot$ day $^{-1}$, mortality rates would have respectively been $16.7 \%$ (21 of 126$), 24.7 \%$ (21 of 85 ), $27.4 \%$ (20 of 73), 30.8\% (20 of 65) and 37.5\% (15 of 40) for the severe group and 5.1\% (2 of 39), $2.5 \%$ ( 2 of 80 ), $3.3 \%$ ( 3 of 92 ), $3 \%$ (3 of 100) and 6.4\% ( 8 of 125) for the moderate group. 
Our study has several limitations. The retrospective design limited the possibility to analyse different therapeutic approaches as more aggressive therapies may have been limited in patients with a poor prognosis due to their underlying disease. Haemoptysis volume and duration may also not have been recorded accurately. While reviewing the charts, we relied on written information about the volume of haemoptysis. No specific measurement tool was routinely used although nursing staff in our institution are instructed to objectively measure haemoptysis in a graded sputum collection container in cases that were moderate to severe. We are aware quantifying moderate-to-severe haemoptysis in the acute scenario can be challenging. Therefore, all reported quantities may not be perfectly accurate, and the presence of recall bias is likely. Our study only analysed data from a single centre which may have induced a selection and reference bias. Finally, the cohort of patients studied is also relatively small, but it was nevertheless larger than other studies of significant haemoptysis, which had fewer than 100 patients $[9-22,28,29]$.

\section{Conclusion}

In conclusion, lung cancer and bronchiectasis were the leading causes of moderate-to-severe haemoptysis in our contemporary North American population. CT and bronchoscopy are complementary to localise the source of bleeding. BAE is effective in the majority of cases at controlling haemoptysis. Haemoptysis of 50-200 mL was associated with a significantly better outcome than haemoptysis $>200 \mathrm{~mL}$.

Conflict of interest: None declared.

\section{References}

1 Khalil A, Fedida B, Parrot A, et al. Severe hemoptysis: From diagnosis to embolization. Diagn Interv Imaging 2015; 96: 775-788.

2 Knott CJ. Management and prognosis of massive hemoptysis. Recent experience with 120 patients. $J$ Thorac Cardiovasc Surg 1993; 105: 394-397.

3 Fartoukh M, Khoshnood B, Parrot A, et al. Early prediction of in-hospital mortality of patients with hemoptysis an approach to defining severe hemoptysis. Respiration 2012; 83: 106-114.

4 Lee MK, Kim SH, Yong SJ, et al. Moderate hemoptysis: recurrent hemoptysis and mortality according to bronchial artery embolization. Clin Respir J 2015; 9: 53-64.

5 Abdulmalak C, Cottenet J, Beltramo G, et al. Haemoptysis in adults: a 5-year study using the French nationwide hospital administrative database. Eur Respir J 2015; 46: 503-511.

6 Gagnon S, Quigley N, Dutau H, et al. Approach to hemoptysis in the modern era. Can Respir J 2017; 2017: 1565030 .

7 Hsiao EI, Kirsch CM, Kagawa FT, et al. Utility of fiberoptic bronchoscopy before bronchial artery embolization for massive hemoptysis. AJR Am J Roentgenol 2001; 177: 861-867.

8 Parrot A, Tavolaro S, Voiriot G, et al. Management of severe hemoptysis. Expert Rev Respir Med 2018; 12: 817-829.

9 Bhalla A, Pannu AK, Suri V. Etiology and outcome of moderate-to-massive hemoptysis: experience from a tertiary care center of North India. Int J Mycobacteriol 2017; 6: 307-310.

10 Ketai LH, Mohammed TL, Kirsch J, et al. ACR appropriateness criteria ${ }^{\circ}$ hemoptysis. J Thorac Imaging 2014; 29: W19-W22.

11 Revel MP, Fournier LS, Hennebicque AS, et al. Can CT replace bronchoscopy in the detection of the site and cause of bleeding in patients with large or massive hemoptysis? AJR Am J Roentgenol 2002; 179: 1217-1224.

12 Haponik EF, Britt EJ, Smith PL, et al. Computed chest tomography in the evaluation of hemoptysis: impact on diagnosis and treatment. Chest 1987; 91: 80-85.

13 Davoodi M, Kordi M, Gharibvand MM, et al. Hemoptysis: comparison of diagnostic accuracy of multi detector CT scan and bronchoscopy. Glob J Health Sci 2015; 7: 373-377.

14 Chalumeau-Lemoine L, Khalil A, Prigent H, et al. Impact of multidetector CT-angiography on the emergency management of severe hemoptysis. Eur J Radiol 2013; 82: e742-e747.

15 Rémy J, Voisin C, Dupuis C, et al. "Traitement des hémoptysies par embolisation de la circulation systémique" [Treatment of hemoptysis by embolization of the systemic circulation]. Ann Radiol (Paris) 1974; 17: 5-16.

16 Ong TH, Eng P. Massive hemoptysis requiring intensive care. Intensive Care Med 2003; 29: 317-320.

17 Coder R. Hemoptysis. Emerg Med Clin North Am 2003; 21: 421-435.

18 Ibrahim WH. Massive heamoptysis: the definition should be revised. Eur Respir J 2008; 32: 1131-1132.

19 Amirana M, Frater R, Tirschwell P, et al. An aggressive surgical approach to significant hemoptysis in patients with pulmonary tuberculosis. Am Rev Respir Dis 1968; 97; 187-192.

20 Corey R, Hla KM. Major and massive hemoptysis: reassessment of conservative management. Am J Med Sci 1987; 294: 301-309.

21 Valipour A, Kreuzer A, Koller H, et al. Bronchoscopy-guided topical hemostatic tamponade therapy for the management of life-threatening hemoptysis. Chest 2005; 127: 2113-2118.

22 Kiral H, Evman S, Tezel C, et al. Pulmonary resection in the treatment of life-threatening hemoptysis. Ann Thorac Cardiovasc Surg 2015; 21: 125-131.

23 Radchenko C, Alraiyes AH, Shojaee S. A systematic approach to the management of massive hemoptysis. J Thorac Dis 2017; 9: Suppl. 10, S1069-S1086.

24 Bhattacharyya P, Dutta A, Samanta AN, et al. New procedure: bronchoscopic endobronchial sealing: a new mode of managing hemoptysis. Chest 2002; 121: 2066-2069.

25 Brandes JC, Schmidt E, Yung R. Occlusive endobronchial stent placement as a novel management approach to massive hemoptysis from lung cancer. J Thorac Oncol 2008; 3: 1071-1072. 
26 Bylicki O, Vandemoortele T, Laroumagne S, et al. Temporary endobronchial embolization with silicone spigots for moderate hemoptysis: a retrospective study. Respiration 2012; 84: 225-230.

27 Kho SS, Chan SK, Yong MC, et al. Endobronchial embolization for life-threatening hemoptysis with Endobronchial Watanabe Spigot. BMC Res Notes 2017; 10: 304.

28 Lee TW, Wan S, Choy DK, et al. Management of massive hemoptysis: a single institution experience. Ann Thorac Cardiovasc Surg 2000; 6: 232-235.

29 Shigemura N, Wan IY, Yu SC, et al. Multidisciplinary management of life-threatening massive hemoptysis: a 10-year experience. Ann Thorac Surg 2009; 87: 849-853. 